Thorax, 1977, 32, 777-780

\title{
Permanent right ventricular pacing through an anomalous left superior vena cava
}

\author{
SHLOMO AMIKAM, JOSEPH LEMER, AND EGON RISS
}

From the Departments of Cardiology and Cardiac Surgery, Rambam Medical Centre, Aba Khoushy School of Medicine, Haifa, Israel

Amikam, S., Lemer, J., and Riss, E. (1977). Thorax, 32, 777-780. Permanent right ventricular pacing through an anomalous left superior vena cava. A persistent left superior vena cava can complicate the implantation of a transvenous pacemaker. In a patient who required a permanent pacemaker, this venous anomaly was discovered during the insertion of the electrode but it did not prevent long-term right ventricular pacing. This was achieved after the electrode had been manipulated through the coronary sinus and right atrium. A plan of management is proposed for dealing with this unexpected problem.

The majority of permanent pacemakers implanted today are of the pervenous endocardial type. In most cases the endocardial electrode is introduced through the cephalic vein in the deltopectoral triangle. One of the problems that may be encountered when using the transvenous endocardial electrode is the presence of a persistent left superior vena cava, which drains directly into the coronary sinus. This problem usually occurs unexpectedly, and, from the few descriptions of the anomaly discovered during pacemaker implantation, it is evident that no definite method of management has been established to deal with such cases (Kukral, 1971; Rose et al., 1971; Garcia et al., 1972; Rubenfire et al., 1974).

The purpose of this paper is to present an additional case of a persistent left superior vena cava (PLSVC) which was found during the introduction of a pervenous permanent endocardial electrode and to describe the manner in which we dealt with this problem. We also propose a plan of management to be applied when faced with PLSVC during the introduction of a transvenous electrode.

\section{Case report}

A 61-year-old woman was diagnosed as suffering from sick-sinus syndrome five years before the present admission. She had episodes of rapid atrial fibrillation and supraventricular tachycardia which produced palpitation, chest pain, and dizziness. Apart from these episodes of tachydysrhythmia her heart rate was slow. In the few weeks before admission the frequency of the bouts of rapid atrial fibrillation increased and the attacks could be terminated only with the aid of antidysrhythmic agents which produced persistent symptomatic sinus or nodal bradycardia. It was decided to treat the patient with a permanent endocardial pacemaker without the initial insertion of a temporary electrode.

At operation a $58 \mathrm{~cm}$ length Medtronic bipolar electrode was introduced via the left cephalic vein into the left subclavian vein. Under fluoroscopic control it was found that the course taken by the electrode was through a persistent left superior vena cava draining into the coronary sinus. The electrode was removed and left subclavian venography was performed, confirming the presence of an anomalous left superior vena cava draining into the coronary sinus without connection to the venous system of the right side. The electrode was again introduced via the left superior vena cava and the coronary sinus into the right atrium and could be manipulated into a right superior vena cava. By further manoeuvring and after the formation of a loop in the right atrium the electrode tip was advanced to the apex of the right ventricle. As fluoroscopy showed adequate impaction of the electrode tip and satisfactory pacing was obtained with a threshold of $1.0 \mathrm{~mA}$, and as the paced QRS complex demonstrated a left bundle-branch block pattern, it was decided to leave the electrode in this position for permanent pacing. The pulse-generator (Medtronic 


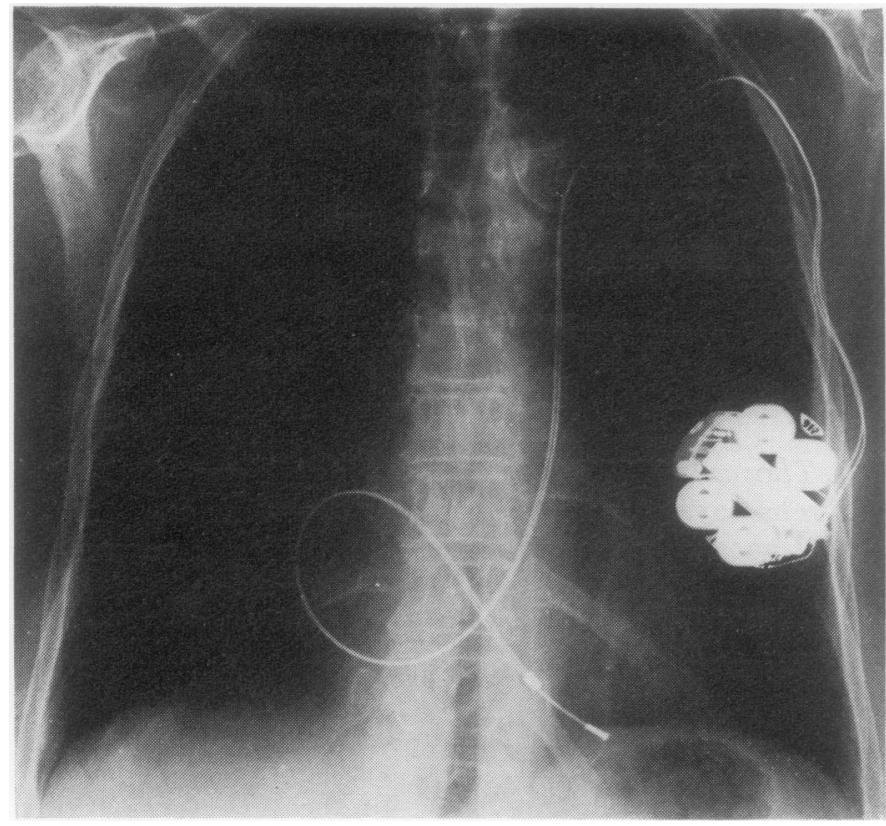

Fig. 1 Posteroanterior chest radiograph showing the transvenous electrode tip in the right ventricle after traversing a left $\mathrm{N}_{\mathrm{N}}$ superior vena cava and the coronary sinus $\sigma$ with the formation of a loop in the right atrium.

demand No. 5950) was connected and implanted in a left subcutaneous pectoral pocket.

Seven days later the patient was discharged, receiving a maintenance dose of $80 \mathrm{mg}$ propranolol daily. A chest radiograph confirmed that the electrode tip had remained lodged in the apex of the right ventricle (Figs. 1 and 2).

Six months later the patient was well and symptomless. Electrocardiographic tracings during routine follow-up examinations showed consistent pacing most of the time.

\section{Discussion}

Persistent left superior vena cava (PLSVC) is estimated to be present in $0.5 \%$ of the general population (Fraser et al., 1961) and is said to occur in 0.2 to $4.3 \%$ of all congenital cardiac anomalies (Campbell and Deuchar, 1954; Shumacker et al., 1967). In a recent review of 100 patients with anomalous systemic venous connections, de Leval et al. (1975) found that the commonest anomaly was a PLSVC which was present in 47 patients. Until recently the importance of this anomaly was mainly the concern of the thoracic surgeon when cannulating the heart for cardiopulmonary bypass or, in the case of transposition of the great arteries, when a Mustard procedure was planned.

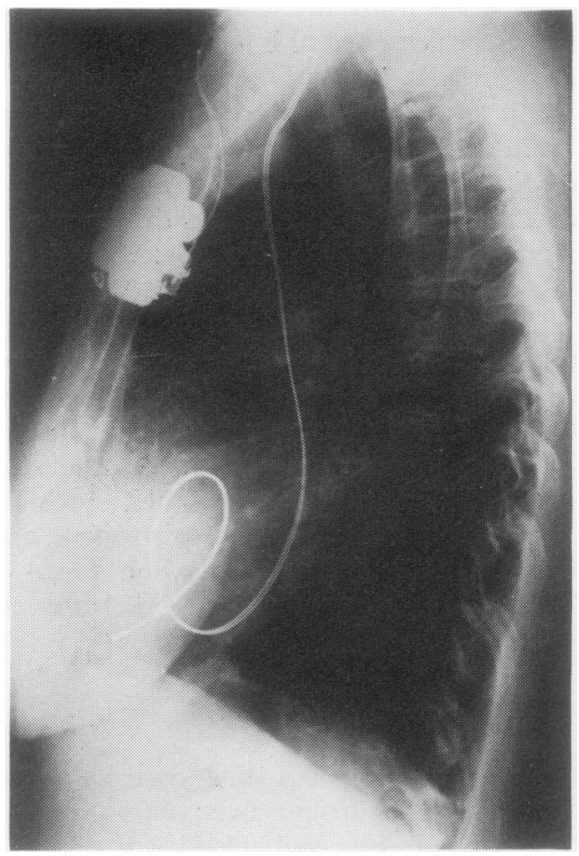

Fig. 2 Lateral chest radiograph showing the unusual course taken by the electrode, which traverses the left superior vena cava, coronary sinus, and right atrium to reach the right ventricle. 
The recent expansion of pacemaker implantation, especially of the transvenous endocardial type, should bring this problem to light more frequently. The physician may unexpectedly face this anomaly in at least one out of 200 endocardial insertions of temporary or permanent pacemakers. We, therefore, feel that a plan of management of the problem should be outlined.

There are a few reports in the literature which describe the discovery of this anomaly during the insertion of a pacemaker electrode. Kukral (1971) described a case where the electrode, which was introduced into the right cephalic vein, took an unusual course but gave initial satisfactory pacing. However, within a few hours pacing ceased, causing the death of the patient. At postmortem examination a PLSVC was discovered draining into the coronary sinus. It is not clear whether pacing was obtained in this case from the coronary sinus or from the right ventricle, and, if from the latter, how entry into the right ventricle was achieved. We assume that the electrode in this case was lodged from the beginning in the coronary sinus. Rubenfire et al. (1974) reported a case in which a permanent electrode was introduced through the left cephalic vein and, after traversing a PLSVC, was lodged in the posterior coronary vein. Although the initial threshold was satisfactory, failure of pacing occurred the same day, requiring the insertion of a temporary pacemaker via the right basilic vein. In this case a right SVC was present and therefore a permanent electrode was subsequently inserted in the normal manner via the right cephalic vein to the right ventricular apex. As in the first case, failure of pacing occurred initially, probably because the clectrode was left in the coronary sinus. Rose et al. (1971) described successful introduction of a permanent electrode into the apex of the right ventricle through a PLSVC system. Their initial attempt at endocardial pacing was via the right cephalic vein using a $58 \mathrm{~cm}$ electrode. As it took an aberrant course and was too short to reach the right ventricle, a diagnostic venogram was performed, confirming the presence of a solitary PLSVC. Later an $85 \mathrm{~cm}$ electrode was inserted by the left cephalic vein via the PLSVC system to the coronary sinus and right atrium and, with the aid of a loop formation, into the right ventricle. In this position, as in our case, long-term endocardial pacing was maintained without difficulty. In a similar case reported by Garc'a et al. (1972), permanent pacing was achieved for 23 months, using the same electrode course described by Rose and by us. However, after that time, when the pulse-generator was replaced because of its failure, it seemed to the surgeon during fluoroscopy that the electrode tip was no longer located within the right ventricle. Attempts at manipulating the electrode tip back into its original position failed. Venography revealed the absence of a right SVC, and therefore epicardial electrodes were implanted via a thoracotomy.

In both our case and the one described by Rose it has been demonstrated that long-term right ventricular pacing can be achieved despite the presence of an anomalous PLSVC. Failure of pacing in the two additional reported cases was probably due to the lodgement of the electrode tip in the coronary sinus. Successful long-term coronary sinus pacing has been reported in several series but only when the electrode was introduced in the normal manner after passage through the right atrium. In the case of PLSVC, the electrode reaches the coronary sinus from the opposite end and, with this approach, long-term pacing can probably not be obtained.

We propose that the following steps should be taken when the operator is faced with this anomaly during endocardial pacemaker implantation. When using the left-sided approach: (1) Perform venography to demonstrate the presence or absence of a venous connection to the right side and a right SVC. In approximately $75 \%$ of these cases there is hypoplasia or agenesis of the left innominate vein (Winter, 1954), and therefore there is no connection to the right side. (2) Advance the electrode through the coronary sinus into the right atrium and, by manipulating it, again determine the presence of a right SVC entering the right atrium. (3) Try to manipulate the tip of the electrode into the apex of the right ventricle. This step can be achieved only with the aid of a loop in the right atrium. Satisfactory threshold measurement is the evidence for good lodgement of the tip. (4) If repeated attempts at entry into the right ventricle fail, and a right SVC was previously demonstrated, the left-sided approach should be abandoned and a formal right-sided approach adopted. (5) If entry to the right ventricle was not achieved and a right SVC is absent, the only remaining possibility is an epicardial implantation.

Faced with a solitary left SVC from an initial right-sided approach: (1) Exchange the $58 \mathrm{~cm}$ electrode for a longer one and attempt a leftsided approach as described above. (2) If this fails, the only possibility remaining is epicardial implantation.

In those patients in whom this anomaly is detected during temporary implantation, elective epicardial insertion should be the obvious solution. 
However, when faced with this anomaly unexpectedly, in our opinion the physician should try to solve the problem in the manner we have described. He should remember that temporary pacing through an anomalous left superior vena cava can also be achieved.

\section{References}

Campbell, M., and Deuchar, D. C. (1954). The leftsided superior vena cava. British Heart Journal, 16, 423-439.

de Leval, M. R., Ritter, D. G., McGoon, D. C., and Danielson, G. K. (1975). Anomalous systemic venous connection. Surgical considerations. Mayo Clinic Proceedings, 50, 599-610.

Fraser, R. S., Dvorkin, J., Rossall, R. E., and Eidem, R. (1961). Left superior vena cava. American Journal of Medicine, 31, 711-716.

Garcia, L., Levine, R. S., Kossowsky, W., and Lyon, A. F. (1972). Persistent left superior vena cava complicating pacemaker catheter insertion. Chest, 61, 396-397.
Kukral, J. C. (1971). Transvenous pacemaker failure due to anomalous venous return to the heart. Chest, 59, 458-461.

Rose, M. E., Gross, L., and Protos, A. (1971). Transvenous pacemaker implantation by way of an anomalous left superior vena cava. Journal of Thoracic and Cardiovascular Surgery, 62, 965-966.

Rubenfire, M., Evangelista, J., Wajszczuk, W. J., and Kantrowitz, A. (1974). Implication of a persistent left superior vena cava in transvenous pacemaker therapy and cardiac hemodynamic monitoring. Chest, 65, 145-147.

Shumacker, H. B., Jr., King, H., and Waldhausen, J. A. (1967). The persistent left superior vena cava: surgical implications, with special reference to caval drainage into the left atrium. Annals of Surgery, 165, 797-805.

Winter, F. S. (1954). Persistent left superior vena cava: survey of world literature and report of thirty additional cases. Angiology, 5, 90-132.

Requests for reprints to: Dr. S. Amikam, Department of Cardiology, Rambam Medical Centre, Bat-Galim, Haifa, Israel. 